\title{
Skyrmions in a Half-Filled Second Landau Level
}

\author{
A. Wójs ${ }^{1,2}$, G. Möller ${ }^{1}$, S. H. Simon ${ }^{3}$, and N. R. Cooper ${ }^{1}$ \\ ${ }^{I}$ TCM Group, Cavendish Laboratory, University of Cambridge, Cambridge CB3 OHE, United Kingdom \\ ${ }^{2}$ Institute of Physics, Wroclaw University of Technology, 50-370 Wroclaw, Poland \\ ${ }^{3}$ Rudolf Peierls Centre for Theoretical Physics, University of Oxford, Oxford OX1 3NP, United Kingdom
}

\begin{abstract}
We studied charged excitations of the $v=5 / 2$ fractional quantum Hall state allowing for spin depolarization. It is generally accepted that the ground state is a spin-polarized incompressible quantum liquid, adiabatically connected to the Pfaffian state, whose spin-polarized quasiholes (QHs) obey non-Abelian statistics. Using numerical diagonalization and taking account of non-zero well widths we demonstrated that at a sufficiently low Zeeman energy it is energetically favorable for pairs of charge e/4 QHs to bind into charge e/2 Skyrmions. We showed that Skyrmion formation is further promoted by disorder, and argue that this can lead to a depolarized ground state in realistic experimental situations.
\end{abstract}

Keywords: Pfaffian, Skyrmion, non-Abelian statistics, fractional quantum Hall effect.

PACS: 73.43.-f, 71.10.Pm

The continued interest in the fractional quantum Hall (FQH) state at the Landau level (LL) filling factor $v=5 / 2[1]$ is motivated by accumulating evidence that it realizes a non-Abelian phase of matter - the Pfaffian (Pf) state defined by Moore and Read [2], which could open the way to fault-protected topological quantum computation [3]. One of the outstanding questions has been, for a long time [4], that of its spin polarization. While the numerical simulations of small systems have consistently pointed to a fully polarized ground state in a half-filled second LL [5], especially the most recent experimental evidence for a partial depolarization at $v=5 / 2[6]$ has also become very convincing. This paper aims at resolving this discrepancy by presenting the numerical results suggesting that the experimentally observed depolarization is due to the topological spin excitations called Skyrmions [7]. Our key prediction is that in the realistic conditions (including small Zeeman spin splitting, nonzero layer thickness, and disorder) at $v=5 / 2$, the charge $\pm \mathrm{e} / 2$ Skyrmions spontaneously form by binding pairs of charge $\pm \mathrm{e} / 4$ quasiholes (QHs) or quasiparticles (QPs) in the polarized ground state.

For the purpose of numerical simulations, we have considered systems of $N$ electrons confined to a sphere and exposed to magnetic flux $N_{\phi}$ [8]. In this geometry, an extended incompressible liquid at filling factor $v$ is represented by a series of nondegenerate ground states at $N_{\phi}=N / v-\sigma$, with a constant "shift" $\sigma$. In the search of an unpolarized $v=1 / 2$ liquid, we diagonalized systems with $N \leq 12$ and different $\sigma$, simultaneously resolving the total spin $S$ and angular momentum $L$. To avoid the known problem of aliasing with the $v=2 / 3$ series [4], we considered fairly large systems, with the Hilbert space dimensions reaching $1.4 \cdot 10^{9}$ (for $N=12, N_{\phi}=26$ ). Cosine subband wave function was assumed, and the effective layer thickness was varied between $w=0$ and $3 \lambda$ ( $\lambda$ being the magnetic length). For a hypothetical unpolarized $v=1 / 2$ liquid, the correlation energy per particle $E$ and the pair correlation function $g(\mathrm{r})$ should depend smoothly on $N$ along the series. Obviously, $E$ should extrapolate (for $1 / N \rightarrow 0$ ) to a lower value than that of the (Pf) polarized ground state at $\sigma=3$.

The results for $E(N, \sigma)$ are shown in Fig. 1. Several series consistently have $L=0$ and appear to extrapolate to the same energy as the Pf series (independently of the layer thickness $w$ ). The values of $\sigma=-2,0,2$, and 4 for these series suggest that they represent global spin textures (Skyrmions) formed in the Pf state and in its particle-hole conjugate called the anti-Pfaffian (APf).

Skyrmions in a ferromagnetic liquid are topological excitations in which the local spin orientation wraps once over the surface of the spin sphere. Coupling of spin and orbital degrees of freedom makes a Skyrmion appear as a single effective flux quantum, leading to a net charge ve [7]. E.g., Skyrmion in the Pf state ( $\left.S^{+} \mathrm{Pf}\right)$

occurs at $\sigma=2$ and carries charge $+\mathrm{e} / 2$. In fact, the four series identified in Fig. 1 correspond to the Skyrmions and anti-Skyrmions formed in the Pf or anti-Pf (APf; particle-hole conjugate of the Pf; $\sigma=-1$ ) ground states.

Skyrmion interpretation of the $S=0$ ground states of Fig. 1 is confirmed by their charge $(g)$ and spin $(f)$ pair correlation functions, compared to the $g(r)$ curves of their polarized parents. The relevant plots are shown in Fig. 2(a). Clearly, $g(r)$ of $S^{+}$Pf and $S^{+}$APf are closely follow those of Pf and APf, respectively, while $f(r)$ decreases smoothly along the entire sphere (except for the irrelevant $r \sim 0$ region of $g \approx 0$ ) for both states. This is 
precisely the behavior expected of the Skyrmions (by analogy with the $v=1$ or $1 / 3$ cases studied previously).

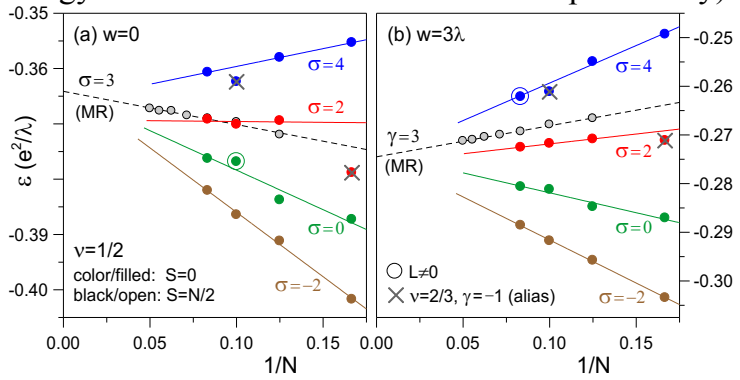

FIGURE 1. Size extrapolation of the ground-state energies per particle $\varepsilon$ of $N$ electrons in a half-filled second Landau level, for layer widths $w=0$ (a) and $3 \lambda$ (b) Competing series of candidate unpolarized ground states are distinguished by their shifts $\sigma$, and compared to the polarized (Pfaffian) series with $\sigma=3$. Crosses - alias states excluded from extrapolation; open circles - two exceptional cases with the marginally excited $L=0$ states; $\lambda$ is the magnetic length.

The $\sigma=2$ ground state of Fig. 1 was also compared with the following model Skyrmion state, explained in Fig. 2(b) for the case of $N=10$. Beginning with the full $L=S=0$ Hilbert space $\mathcal{H}($ dimension $d=1581)$, we first

construct a zero-energy subspace $\mathcal{H}^{\prime}(d=105)$ of the pair interaction with a single pseudopotential, $V_{0}(0)$ [pair pseudopotential $V_{S}(m)$ is defined as the energy of a two-electron state with spin $S$ and relative angular momentum $m$ ]. Next, within $\mathcal{H}^{\prime}$ we apply the triplet interaction with only a single pseudopotential, $W_{3 / 2}(3)$ [triplet pseudopotential $W_{S}(m)$ is the energy of the three-electron state with spin $S$ and relative angular momentum $m$ ] to obtain a zero-energy subspace $\mathcal{C}^{\prime \prime}$

$(d=21)$ containing the unpolarized states which retain the Pf-like correlations at short range. Finally, inside $\mathcal{H}$ " we use $V_{0}(2)$ to select as the lowest state the one with the longest spin wave length, i.e., the smooth global spin texture (Skyrmion) formed in an exact Pf parent. Remarkably, the only significant drop in the squared projections $|\chi|^{2}$ of the Coulomb ground states onto the succesively reduced subspaces occurs on the action of $W$. Hence, the moderate values of $|\chi|^{2}=0.53$ and 0.76 (for $w=0$ and $3 \lambda$ ) at this step are most likely inherited from the known, similarly moderate squared overlaps of the Coulomb polarized ground state with the exact Pfaffian wave functions.

Can these Skyrmions be stable? Carrying twice the charge of an elementary spinless excitation, they must combine two QHs or QPs, overcoming their Coulomb repulsion. Careful comparison with the energies of two

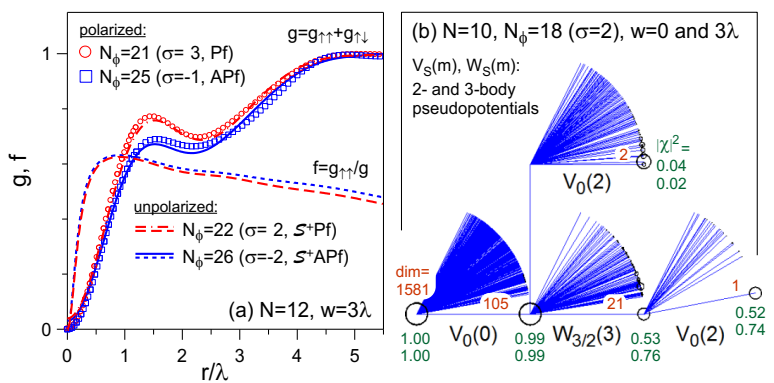

FIGURE 2. (a) Charge and spin pair-correlation functions ( $g$ and $f$ ) of the 12-electron unpolarized $v=1 / 2$ ground states with $\sigma=2$ and -2 , compared to their spin-polarized (Pfaffian and anti-Pfaffian) parents. (b) Splitting of the $L=S=0$ Hilbert space for $N=10, v=1 / 2$, and $\sigma=2$ under the consecutive action of the pair and triplet pseudopotentials, $V_{S}(m)$ and $W_{S}(m)$. At each step, the green labels give squared projections of the Coulomb ground state onto the lowest subspace (upper and lower value are for $w=0$ and $3 \lambda$ ) with dimension indicated in red. The nondegenerate ground state obtained by successive application of $V_{0}(0), W_{3 / 2}(3)$, and $V_{0}(2)$ is an approximation to the exact Skyrmion formed in the Pfaffian ground state.

distant QPs or QHs (including electrostatic corrections [9]) confirms stability of the positively charged $S^{+} \mathrm{Pf}$ and $\mathcal{S}$ APf in sufficiently wide layers $(w \geq \lambda)$. Stability of the negatively charged $S_{\text {Pf }}$ or $S^{+}$APf states is also possible, but it requires sufficiently strong disorder [10] (helping to bring two QPs against their repulsion) in addition to the sufficiently small Zeeman splitting.

In conclusion, the QHs or QPs of the $v=5 / 2$ ground state can, in realistic experimental conditions (nonzero layer thickness, small Zeeman splitting, disorder) bind into Skyrmions, causing depolarization of spin.

This work has been supported by EU under Marie Curie Grant No. PIEF-GA-2008-221701 (AW), Trinity Hall (GM); and EPSRC under EP/F032773/1 (NRC).

\section{REFERENCES}

1. R. Willett et al., Phys. Rev. Lett. 59, 1776 (1987).

2. G. Moore and N. Read, Nucl. Phys. B 360, 362 (1991).

3. C. Nayak et al., Rev. Mod. Phys. 80, 1083 (2008).

4. R. H. Morf, Phys. Rev. Lett. 80, 1505 (1998).

5. E. H. Rezayi and F. D. M. Haldane, Phys. Rev. Lett. 84, 4685 (2000); A. Feiguin et al., Phys. Rev. B 79, 115322 (2009).

6. J. P. Eisenstein et al., Phys. Rev. Lett. 61, 997 (1988); C. R. Dean et al., ibid. 100, 146803 (2008); 101, 186806 (2008); T. D. Rhone and A. Pinczuk (unpublished).

7. S. L. Sondhi et al., Phys. Rev. B 47, 16419 (1993); N. R. Cooper, Phys. Rev. B 55, 1934 (1997); A. H. MacDonald and J. J. Palacios, ibid. 58, 10171 (1998).

8. F. D. M. Haldane, Phys. Rev. Lett. 51, 605 (1983).

9. R. H. Morf et al., Phys. Rev. B 66, 075408 (2002).

10. A. Wójs et al., Phys. Rev. Lett. 104, 086801 (2010). 